\title{
Sesamol ameliorates acute kidney injury resulting from complete unilateral ureteral obstruction in the rats
}

\author{
UGUR UYETURK ${ }^{1}$, AYHAN ÇETINKAYA ${ }^{2}$, EMRE BOSTANCI ${ }^{1}$, SEVILAY ERIMSAH ${ }^{3}$, HAYRETTIN OZTURK ${ }^{4}$ \\ ${ }^{1}$ Bolu Abant Izzet Baysal University, Faculty of Medicine, Department of Urology, Bolu, Turkey uguruyeturk@yahoo.com , \\ dremrebostanci@gmail.com \\ ${ }^{2}$ Bolu Abant Izzet Baysal University, Faculty of Medicine, Department of Physiology, Bolu, Turkey cetinkayaayhan@hotmail.com \\ ${ }^{3}$ Bolu Abant Izzet Baysal University, Faculty of Medicine, Department of Histology and Embryology, Bolu, Turkey \\ sevilayerimsah@yahoo.com \\ ${ }^{4}$ Bolu Abant Izzet Baysal University, Faculty of Medicine, Department of Pediatric Urology, Bolu, Turkey ozturkhayrettin@hotmail.com \\ Corresponding Author: Prof. Dr. Hayrettin Ozturk, Bolu Abant Izzet Baysal University, Faculty of Medicine, Department of Pediatric Urology, \\ Bolu, Turkey, Email: ozturkhayrettin@hotmail.com, Phone: 0903742534656
}

\begin{abstract}
Objectives: We aimed to evaluate the effectiveness of sesamol in preventing the progressive renal damage caused by complete unilateral ureter obstruction (CUUO).

Methods: Twenty four Wistar rats were separated into three groups: In the group 1 rats (the sham-control, $\mathrm{n}=8$ ), the ureters were exposed and manipulated, but not ligated. In the group 2 rats (CUUO-untreated, $n=8$ ), CUOO was accomplished by double-ligating the left ureter using 5-0 silk ties. In the group 3 rats (CUUO -Sesamol treated, $n=8$ ), CUOO was created as in the Group 2 rats, also given sesamol daily, commencing on the day of CUUO surgery. The rats' left kidneys were examined histologically and biochemically within the $8^{\text {th }}$ day

Results: Histopathological total score, apoptotic index and MDA values in the group 2 show a significant increase when compared to the group 1 . These values were significantly decreased in the group 3 rats when compared to the group 2 rats. Tissue GPx value in the group 2 show a significant decrease when compared to Sham-control group. However, these values were significantly increased in the group 3 rats when compared to the rats 2. Polymorph nuclear leukocyte infiltration in peritubular areas was observed in group II, with moderate interstitial fibrosis and tubular dilatation. Despite the presence of dilated tubule structures in group III, this group showed less bleeding, leukocyte infiltration, and fibrosis than group II.

Conclusion: The results of this study have shown that CUOO-induced renal damage can be reduced using sesamol, possibly due to its antioxidant properties. The mechanism by which sesamol causes these effects merits further investigation.
\end{abstract}

Keywords: Complete unilateral ureteral obstruction, renal damage, sesamol, oxidative stress, rat.

\section{INTRODUCTION}

In adult humans, the most common cause of unilateral acute ureteral obstruction is ureteral stones, causing loss of kidney function if the obstruction is not removed early $(1,2)$. Obstructive uropathy causes tubular dilatation, decreased renal perfusion, interstitial inflammation, apoptosis and necrosis, and when it is progressive, it triggers tubular cell death due to fibrosis $(1,2)$. Therefore, preserving kidney function in the acute phase is invaluable. In addition, some studies have suggested that the detrimental effect of obstructive nephropathy may persist even after the obstruction is resolved (3).

Clinical and experimental studies have suggested that oxidative stress plays an important role in the pathogenesis of obstructive uropathy $(1,4)$. Oxidative stress results in excessive ROS production and / or disturbances in cellular antioxidant systems (5). Reactive oxygen species (ROS) derived from the mitochondrial electron transport chain are essential for cell homeostasis, cell survival, and gene expression (6). In addition, ROS is known to induce both mitochondrial apoptosis pathway and apoptosis causing endoplasmic reticulum stress response in obstructed kidneys (7). ROS levels increase from the early stage, whereas the levels of protective antioxidant enzymes, including superoxide dismutase, catalase, and glutathione, decrease. Reactive oxygen scavenging agents significantly reduce renal damage in subjects with obstructive kidney disease (8).
Sesame (Sesamumindicum L., family Pedaliaceae) grows in mild climates (9). It is mainly used to delay aging and to treat diseases in Eastern medicine. Sesame seeds mainly contain sesamolin, sesamin, and sesamol (10). Sesamol (3,4-methylenedioxyphenol) is a free radical scavenging compound with antioxidant properties due to the benzodioxole group's presence, which helps maintain the stability of sesame oil. Sesamol has been used in experimental therapies for cardiovascular disorders, pulmonary inflammation, diabetic nephropathy, diabetes mellitus, and neurodegenerative disorders. It has also been demonstrated to have anti-candidal, hepatoprotective, radio-protective, and anti-aging properties (11).

Unilateral acute complete ureteral obstruction (CUUO) is a useful model for investigating the renal response to urinary tract obstruction and treatment alternatives. No study has yet addressed the effectiveness of sesamol in reducing the renal damage associated with CUUO. In this study, we aimed to investigate the effectiveness of sesamol, a free radical scavenger compound with antioxidant properties, in preventing CUOO-induced acute kidney injury, which is frequently seen in urology practice in an experimental CUUO model in rats.

\section{MATERIALS AND METHODS}

Experimental animals: Twenty-four male, Spraguee Dawley rats $(230-250 \mathrm{~g})$ were used in this study, which 
were obtained from the Bolu Abant Izzet Baysal University (BAIBU) (Bolu, Turkey) Animal Care and Research Laboratories. This study was conducted at the Laboratory Animals Application and Research Center at Abant Izzet Baysal University, following official approval by the university's Ethics Board for Animal Studies, (date/ number: 15.11.2017/49). Routine animal care guidelines and the Guide to the Care and Use of Laboratory Animals (1996) were essential in the practice of this study's procedures. All rats were kept in average room temperature $\left(22 \pm 2{ }^{\circ} \mathrm{C}\right)$, $40-70 \%$ humidity for 12 hours in a bright, and then 12 hours in a dark environment. Rats were fed with the standard palette (210 kcal $\backslash 100$ glday) and tap water. All of the rats were first placed on a homeothermic table to maintain a 37 _C body temperature. Anesthesia was administered with an intraperitoneal injection of xylazine $(10 \mathrm{mg} / \mathrm{kg})$ and ketamine hydrochloride (100 mg/kg of Ketalar). Following a right femoral venous catheterization, fluid replacement was performed during the process with $3 \mathrm{~mL} \mathrm{~kg} 1 \mathrm{~h} 1$ of Lactated Ringer solution by using an infusion pump.

Experimental protocol and surgical procedures: In accordance with the procedure previously described in the literature, CUUO was also completed [1-3]. After general anesthesia with ketamine and xylazine, complete ureteral obstruction was accomplished by double-ligating the left ureter using 5-0 silk ties following an abdominal incision. In sham-operated rats, the ureters were exposed and manipulated, but not ligated. Following the procedure, the incision was closed in the usual manner using $3 / 0$ Vicryl.

In our study, the control and CUUO groups were followed for 7 days. In the group 1 rats (the sham-control, $n$ $=8$ ), the ureters were exposed and manipulated, but not ligated. The incision was closed in a single layer. In the group 2 rats (CUUO-untreated, $n=8$ ), CUOO was accomplished by double-ligating the left ureter using 5-0 silk ties. The rats were not given any treatment. In the group 3 rats (CUUO -Sesamol treated, $n=8$ ), CUOO was created as in the Group 2 rats, also given sesamol daily, commencing on the day of CUUO surgery. A $50-\mathrm{mg} / \mathrm{kg}$ dose of sesamol (98\%; no.S3003; Sigma-Aldrich Merck $\mathrm{KGaA}$, Darmstadt, Germany) was diluted in saline (2 $\mathrm{ml})$ and administered by gavage. All of the i.g. administrations were performed after a 12 hour fast, and rats were allowed free access to food $2.5 \mathrm{~h}$ after the procedure (12).

On the eighth day of the study, the all animals were sacrificed by intracardiac puncture under anesthesia, and the left kidneys were excised, and decapsulated for histolpathologic and biochemically examinations. Half the kidney was snap frozen in liquid nitrogen at $-80^{\circ} \mathrm{C}$. The other half was fixed in $10 \%$ neutral-formalin and embedded with paraffin and sectioned $(4 \mu \mathrm{m})$ on a microtome for light microscopy.

Histological Examination: Kidney tissues were placed in $10 \%$ neutral buffered formalin and paraffin-embedded. The paraffin blocks were sectioned to $4 \mu \mathrm{m}$ thickness. The sections were stained with hematoxylin, eosin, and Masson's trichrome to observe the tissue's histological structure under a Nikon (Tokyo, Japan) Eclipse 80 I microscope with NIS-Elements D 3.2 software. Based on the previously published studies, tubular dilatation (TD), interstitial inflammatory cell infiltration (IICI), and interstitial fibrosis (IF) were assessed. Kidney sections were semi- quantitatively evaluated to score all criteria. The severity of obstructive damage to kidneys was scored by percentage, ranging from 0 to 3 (no alteration 0 , mild alteration 1, moderate alteration 2, and severe alteration 3) (13). Histopathological scores were computed for any criteria, and the histopathological total score was calculated by adding all these scores. In order to detect DNA fragmentation in apoptotic cells, TdT-mediated dUTP nick end labeling (TUNEL; Millipore ApopTag® Plus Peroxidase In Situ Apoptosis Detection Kit) method was performed. The renal sections were examined under a light microscope at 10X magnification. The apoptotic index was determined as the number of positive cells per area. A histologist, who blinded to group assignment, evaluated all kidney sections.

Biochemical Evaluation: Tissue samples were minced, placed in phosphate-buffered saline (PBS; pH 7.4), and stored on ice until the blood was separated. Ultrasonic cell lysis was used to prepare the tissue homogenates. Tissue pieces were homogenized in $1 \mathrm{~g}: 9 \mathrm{ml}$ PBS on ice for $5 \mathrm{~min}$ and then centrifuged at $4^{\circ} \mathrm{C}$ at $5000 \times g$ for $5 \mathrm{~min}$. The supernatant was collected and analyzed using the appropriate commercial enzyme-linked immunosorbent assay (ELISA) kit, following the manufacturer's protocol.

Quantitative identification of glutathione peroxidase (GPx, U/g protein) and malondialdehyde (MDA, nmol/g protein) was performed using ELISA kits (catalog \#s E-ELH5410 96T and E-EL-0060 96T, respectively; Elab Science, Bethesda, MD, USA) based on the sandwich ELISA principle.

Statistical Evaluation: Statistical analyses were conducted using SPSS software (v. 20; SPSS Inc., Chicago, IL, USA). The conformity of the variables to normal distribution was examined visually (using histograms and probability graphs) and analytically (using the Kolmogorov-Smirnov and/or Shapiro-Wilk tests). Descriptive analyses were conducted using the means and standard deviations of normally distributed variables. MDA and apoptotic cell counts were compared between groups using a one-way analysis of variance, as these parameters were normally distributed. The homogeneity of variances was evaluated with the Levene test. $P$ values $<0.05$ were considered to be statistically significant. Post-hoc pairwise comparisons were made to detect differences using Tamhane's T2 test. Descriptive analyses were conducted with medians and interquartile ranges of non-normally distributed variables with Bonferroni correction.

\section{RESULTS}

The mean histopathological total score, apoptotic index, MDA and GPx values of the groups are given in Table 1 and Figure 1. Apoptotic index, total score, MDA, GPx were significantly different between the groups (for all $p<0.001$ ). Histopathological total score, apoptotic index and MDA values in the group 2 show a significant increase when compared to the group $1(p<0.001, p<0.001$ and $p<$ 0.001 , respectively). However, these values were significantly decreased in the group 3 rats when compared to the group 2 rats $(p<0.045, p<0.001$ and $p<0.001$, respectively). Tissue GPx value in the group 2 show a significant decrease when compared to Sham-control group $(p<0.001)$. However, these values were significantly 
increased in the group 3 rats when compared to the rats 2 $(p<0.007)$.

A normal kidney histological structure was observed in the sham group (Figure 2A). Polymorph nuclear leukocyte infiltration in peritubular areas was observed in group II, with moderate interstitial fibrosis and tubular dilatation when compared with the sham group. Tubular atrophy and bleeding were seen in several areas (Figure 2B). Despite the presence of dilated tubule structures in group III, this group showed less bleeding, leukocyte infiltration, and fibrosis than did group II (Figure 2C).

Table 1. Histopathological and oxidative stress associated parameters in the renal tissues of the groups.

\begin{tabular}{|l|l|l|l|l|}
\hline Groups & Histopathological total score & Apoptotic index & $\begin{array}{l}\text { MDA } \\
\text { nmol/g protein }\end{array}$ & $\begin{array}{l}\text { Gpx } \\
\mathrm{U} / \mathrm{g} \text { protein }\end{array}$ \\
\hline Group 1 $(\mathrm{n}=8)$ & $0.506 \pm 0.15$ & $1.633 \pm 0.61$ & $1.046 \pm 0.005$ & $0.658 \pm 0.02$ \\
\hline Group 2 $(\mathrm{n}=8)$ & $5.546 \pm 0.29 \mathbf{\Delta}$ & $12.533 \pm 2.95 \mathbf{\Delta}$ & $2.143 \pm 0.12 \mathbf{\Delta}$ & $0.363 \pm 0.01 \&$ \\
\hline Group 3 $(\mathrm{n}=8)$ & $3.975 \pm 0.220 \dagger$ & $9.483 \pm 1.29 \dagger$ & $1.541 \pm 0.03 \dagger$ & $0.420 \pm 0.02 \S$ \\
\hline
\end{tabular}

CUUO: Complete unilateral ureter obstruction. Group 1: sham-control. Group 2: CUUO-untreated. Group 3: CUUO Sesamol treated. MDA: Malondialdehyde, GPx: Glutathione peroxidase,

$\Delta$ Histopathological total score, apoptotic index and MDA values in the group 2 show a significant increase when compared to the group 1 ( $p<0.001, p<0.001$ and $p<0.001$, respectively). $\dagger$ However, these values were significantly decreased in the group 3 rats when compared to the group 2 rats $(p<0.045, p<0.001$ and $p<0.001$, respectively).

\& Tissue GPx value in the group 2 show a significant decrease when compared to Sham-control group ( $p<0.001)$. $\S$ However, these values were significantly increased in the group 3 rats when compared to the rats 2 ( $p<0.007)$.

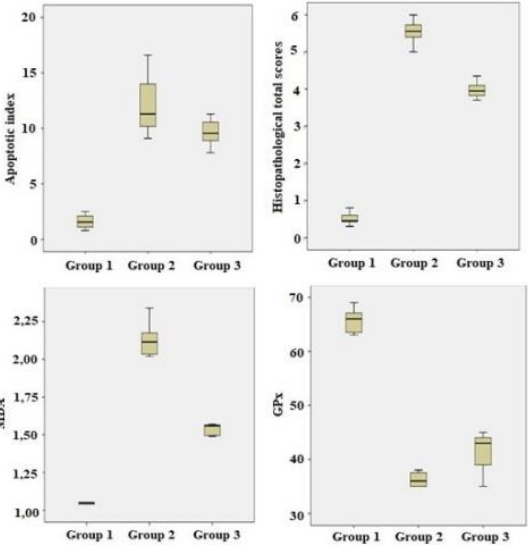

Figure 1. Histopathological and oxidative stress associated parameters in the renal tissues of the groups. CUUO: Complete unilateral ureter obstruction. Group 1: sham-control. Group 2: CUUO-untreated. Group 3: CUUO -Sesamol treated.

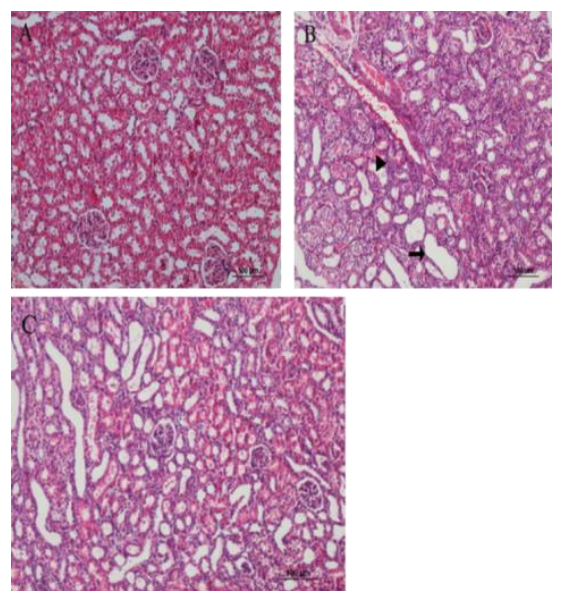

Figure 2. (A) Group 1 (Sham-control); a normal and peritubular stromal tissue is shown (HE, scale bar $100 \mu \mathrm{m}$ ). (B) Group 2 (CUUO-untreated); tubular dilatation, interstitial fibrosis, and in peritubular areas, polymorph nuclear leukocyte (PMN) infiltration (shown as arrow heads) and bleeding is shown (HE, scale bar $100 \mu \mathrm{m}$ ). (C) Group 3 (CUUO -Sesamol treated); In addition to the dilated tubules, reduced fibrosis and $\mathrm{PMN}$ infiltration compared to the Group II is shown (HE, scale bar $100 \mu \mathrm{m}$ ).

\section{DISCUSSION}

Obstruction may occur at any level of the urinary tract, inhibiting urinary flow. This may cause obstructive uropathy and result in renal dysfunction. The frequency and causes of obstruction vary by age and sex. Although stenosis caused by congenital abnormalities is the most common reason for obstruction in the urethra or ureterovesical junction in children, such obstruction is often caused by stones in young adults. In older patients, prostate hypertrophy or retroperitoneal or genital cancer can lead to obstruction (14).

Acute obstruction of the upper urinary tract causes reductions in renal blood flow and glomerular filtration. Intestinal inflammation gradually increases from the early stage and is one of the first tissue responses at the cellular level. Macrophages activated by a variety of cytokines and chemokines participate in this condition. The activated macrophages damage tissues and contribute to tubular apoptosis and interstitial fibrosis (15). Apoptosis plays a critical role in UUO-related tubular atrophy. Tubular distension is a major contributing factor to tubular apoptosis, as well as the resulting oxidative damage (16). Reduction of the levels of endogenous anti-oxygenases increases the apoptosis rate, in contrast to exogenous antioxidant effects (17). Findings from studies indicate that loss of tissue and function due to UUO occurs as a result of a complex process that includes oxidative damage. The effectiveness of Rhodiola Rosea in reducing UUO-related kidney damage was investigated in tissue samples. The results showed that it reduced obstruction-related renal injury. Researchers have concluded that this might be related to the antioxidant property of $\mathrm{Rr}$ (18). Again, in a different study conducted on this subject, the effectiveness of troxerutin was investigated, and in this study, the researchers showed that antioxidative property might decrease kidney damage by limiting inflammation and apoptosis (19). In our study also, the apoptosis in the group in which sesamol was given, decreased compared to the group that was not. However, this did not reach a statistically significant level. 
Ugur Uyeturk, Ayhan Çetinkaya, Emre Bostanci et al

In our study, we observed that there was polymorph nuclear leukocyte (PMN) infiltration in the peritubular areas with interstitial fibrosis and tubular dilatation in the kidneys due to obstruction. Peritubular fibrosis and leukocyte infiltration were detected with dense fibroblastic proliferation in kidney tissue. It was observed that fibrosis and leukocyte infiltration were decreased in the sesamol treated groups compared to those treated without sesamol. We associated this improvement in histological parameters with the protective effect of sesamol on the tissue. They used similar histological parameters in a study to evaluate the efficacy of Nicorandil in reducing UUO-induced kidney damage. They concluded that the antioxidant molecule reduces the degeneration of tubular epithelium and fibrosis, which indicates Nicorandil's effectiveness (20).

Lipids are significantly influenced by oxidative-stressrelated tissue injury. MDA is one of the final products of the peroxidation of polyunsaturated fatty acids. Thus, it is commonly used to evaluate tissue injury. Increases in the MDA level resulting from increased oxidative injury can be ameliorated using exogenous agents, which reduce tissue damage (21). Glutathione-dependent enzymatic antioxidants play a protective role against oxidative damage to tissues. GPx uses glutathione as a co-factor and reduces $\mathrm{H} 2 \mathrm{O} 2$ and organic hydroperoxides (22). Because of such properties, MDA and GPx are used to identify oxidative stress in patients with obstructive nephropathy and resulting tissue injury. GPx decreases as MDA increases (23). In the present study, the MDA levels noticeably increased in other groups compared to the sham group. However, this increase was less in the group in which sesamol was given. Consistent with this, the GPx level was highest in the sham group while the lowest was in the group without sesamol. This showed that ROS increased due to UUO. However, sesamol limited this increase with its antioxidative effect but could not eliminate it.

Various authors have described the therapeutic effectiveness of sesamolin regarding the reduction of tissue injury. One study examined the effectiveness of intraperitoneally administered sesamol in reducing damage to the hematopoietic system, where the injury caused by exposure to ionizing radiation. The authors concluded that sesamol has the potential to protect the hematopoietic system by reducing radiation-based DNA damage and protecting against hematopoietic syndrome (24). Another experimental study evaluated the effectiveness of sesamol in preventing ischemic-reperfusion-related brain damage. The study demonstrated that sesamol has the neuroprotective effects of decreasing oxidative stress and apoptosis, suggesting that sesamol is a potent adjuvant in the treatment of focal cerebral ischemic-reperfusion injury (25). In the current study, we investigated the effectiveness of sesamol in preventing UUO-related renal damage. In the studies evaluating the effect of the molecule on tissues, sesamol was administered with different methods and at different doses. Jan et al. found that $50 \mathrm{mg} / \mathrm{kg}$ orally sesamol was absorbed rapidly in the stomach, and jejunal level and a high rate of it was transferred to kidney tissue (10). In the present study, we preferred to administer sesamol orally and use similar doses to reach an effective tissue level. Our administration method can be applied in clinical conditions much easier than other methods. We observed that oral administration of sesamol might be an effective method to reduce kidney tissue damage considering the histological and biochemical improvement in sesamol groups.

In our study, it was seen that sesamol provided a significant improvement in histological parameters compared to the group not given, and in the biochemical evaluation GPX increased and MDA decreased. Despite this improvement in parameters, there was a statistically significant difference with the sham group. In our study, we preferred to give sesamol $50 \mathrm{mg} / \mathrm{kg}$ orally. We planned the duration of obstruction as seven days. However, we think that not giving different doses and not including different obstruction periods may be a deficiency in our study, and long-short-term modeling and follow-up may contribute more to evaluating the effectivity of the molecule.

\section{REFERENCES}

1. Ayyildiz SN, Ayyildiz A, Benli E, Cirrik S, Germiyanoğlu RC, Noyan T, Çirakoğlu A. The effect of trimetazidine on renal oxidative stress in partial and complete ureteral obstructions in a rat model. Turk J Med Sci. 2017;47(3):1012-1018.

2. Wen JG, Frøkiaer J, Jørgensen TM, Djurhuus JC. Obstructive nephropathy: an update of the experimental research. Urol Res. 1999;27(1):29-39.

3. $S, X u H$, Zou L, Xu G, Wu Z, Ding Q, Jiang $H$. Estrogen preserves split renal function in a chronic complete unilateral ureteral obstruction animal model. Exp Ther Med. 2014 ;7(6):1555-1562.

4. Alfadda AA, Sallam RM. Reactive oxygen species in health and disease. J Biomed Biotechnol 2012; 2012: 936486.

5. Kim JI, Noh MR, Kim KY, Jang HS, Kim HY, Park KM. Methionine sulfoxide reductase A deficiency exacerbates progression of kidney fibrosis induced by unilateral ureteral obstruction. Free Radic Biol Med. 2015;89:201-8.

6. Liu D, Liu Y, Xia Z, Dong H, Yi Z. Reactive oxygen species modulator 1 regulates oxidative stress and induces renal and pulmonary fibrosis in a unilateral ureteral obstruction rat model and in HK-2 cells. Mol Med Rep. 2017;16(4):48554862.

7. Yeh $\mathrm{CH}$, Chiang HS, Lai TY, Chien CT. Unilateral ureteral obstruction evokes renal tubular apoptosis via the enhanced oxidative stress and endoplasmic reticulum stress in the rat. Neurourol Urodyn. 2011;30(3):472-9.

8. Ozbek E. Induction of oxidative stress in kidney. Int $\mathrm{J}$ Nephrol. 2012;2012:465897.

9. Wan Y, Li H, Fu G, Chen X, Chen F, Xie M. The relationship of antioxidant components and antioxidant activity of sesame seed oil. J Sci Food Agric. 2015;95(13):2571-8.

10. Jan KC, Ho CT, Hwang LS. Bioavailability and tissue distribution of sesamol in rat. J Agric Food Chem. 2008;56(16):7032-7.

11. Khan S, Choudhary S, Kumar A, Tripathi AM, Alok A, Adhikari JS, Rizvi MA, Chaudhury NK. Evaluation of sesamol-induced histopathological, biochemical, haematological and genomic alteration after acute oral toxicity in female C57BL/6 mice. Toxicol Rep. $2016 ; 3: 880-894$.

12. Hemalatha G, Pugalendi KV, Saravanan R. Modulatory effect of sesamol on DOCA-salt-induced oxidative stress in uninephrectomized hypertensive rats. Mol Cell Biochem. 2013;379(1-2):255-65.

13. Sancak EB, Turkon H, Cukur S, Erimsah S, Akbas A, Gulpinar MT, et al. Major Ozonated Autohemotherapy Preconditioning Ameliorates Kidney Ischemia-Reperfusion Injury. Inflammation. 2016;39(1):209-17. 
14. Meola M, Samoni S, Petrucci I, Ronco C. Clinical Scenarios in Acute Kidney Injury: Post-Renal Acute Kidney Injury. Contrib Nephrol. 2016;188:64-8.

15. Sean Eardley K, Cockwell P. Macrophages and progressive tubulointerstitial disease. Kidney Int. 2005;68(2):437-55.

16. Chevalier RL. Pathogenesis of renal injury in obstructive uropathy. Curr Opin Pediatr. 2006;18(2):153-60.

17. Sunami R, Sugiyama $H$, Wang $D H$, Kobayashi M, Maeshima Y, Yamasaki Y, et al. Acatalasemia sensitizes renal tubular epithelial cells to apoptosis and exacerbates renal fibrosis after unilateral ureteral obstruction. American journal of physiology Renal Physiol. 2004;286(6):F1030-8.

18. Uyeturk U, Terzi EH, Kemahli E, Gucuk A, Tosun M, Cetinkaya A. Alleviation of kidney damage induced by unilateral ureter obstruction in rats by Rhodiola rosea. $\mathrm{J}$ Endourol. 2013;27(10):1272-6.

19. Kaeidi A, Taghipour Z, Allahtavakoli M, Fatemi I, Hakimizadeh E, Hassanshahi J. Ameliorating effect of troxerutin in unilateral ureteral obstruction induced renal oxidative stress, inflammation, and apoptosis in male rats. Naunyn Schmiedebergs Arch Pharmacol. 2020;393(5):879888.

20. Ozturk H, Firat T, Tekce BK, Yilmaz F, Ozturk H. Effects of nicorandil on renal function and histopathology in rats with partial unilateral ureteral obstruction. Kaohsiung $\mathrm{J}$ Med Sci. 2017;33(5):236-245.

21. Chi KK, Zhang WH, Wang GC, Chen Z, He W, Wang SG, et al. Comparison of Intraperitoneal and Intraepididymal Quercetin for the Prevention of Testicular Torsion/Detorsioninduced Injury. Urology. 2017;99:106-11.

22. Robaczewska J, Kedziora-Kornatowska K, Kozakiewicz M, Zary-Sikorska E, Pawluk H, Pawliszak W, Kedziora J. Role of glutathione metabolism and glutathione-related antioxidant defense systems in hypertension. J Physiol Pharmacol. 2016;67(3):331-7.

23. Dendooven A, Ishola DA, Jr., Nguyen TQ, Van der Giezen DM, Kok RJ, Goldschmeding R, et al. Oxidative stress in obstructive nephropathy. Int J Exp Pathol. 2011;92(3):202-10.

24. Kumar A, Choudhary S, Adhikari JS, Chaudhury NK. Sesamol ameliorates radiation induced DNA damage in hematopoietic system of whole body $\mathrm{Y}$-irradiated mice. Environ Mol Mutagen. 2018;59(1):79-90.

25. Gao XJ, Xie GN, Liu L, Fu ZJ, Zhang ZW, Teng LZ. Sesamol attenuates oxidative stress, apoptosis and inflammation in focal cerebral ischemia/reperfusion injury. Exp Ther Med. 2017;14(1):841-847 